\title{
Analysis of Device-to-Device Communication over Double-Generalized Gamma Channels
}

\author{
Zakir Hussain \\ National University of \\ Computer and Emerging \\ Sciences (NUCES) \\ Karachi, Pakistan
}

\author{
Asim ur Rehman Khan \\ National University of \\ Computer and Emerging \\ Sciences (NUCES) \\ Karachi, Pakistan
}

\author{
Haider Mehdi \\ National University of \\ Computer and Emerging \\ Sciences (NUCES) \\ Karachi, Pakistan
}

\author{
Syed M. Atif Saleem \\ National University of \\ Computer and Emerging \\ Sciences (NUCES) \\ Karachi, Pakistan
}

\begin{abstract}
In this paper, performance of a device-to-device (D2D) communication system is analyzed over double-generalized Gamma (dGG) fading channels. The dGG is a generic distribution for modeling double-scattering fading conditions. Co-channel interference (CCI) caused by various wireless devices in the system is also considered. The CCI fading channel is assumed to be Nakagami distributed. Analytical expressions for important statistical metrics, i.e. probability density function (PDF) and cumulative distribution function (CDF) of signal-tointerference ratio (SIR), are presented. Based on these statistical parameters, expressions for the outage probability, channel capacity and symbol error rate (SER) of the D2D communication system are presented. The performance of D2D system is then discussed and analyzed with the help of numerical results with arbitrary channel fading, path-loss and interference conditions.
\end{abstract}

Keywords-channel capacity; device-to-device communication; double-generalized gamma; outage probability; signal-tointerference ratio; symbol error rate

\section{INTRODUCTION}

The increased growth of smart phones and tablets having many multimedia applications and large file sharing are the motivations for the rapid development in cellular communication systems. These advancements in smart devices with the bandwidth consuming applications have led to increase in demand for higher data rates and this demand is projected to grow exponentially in the future. One of the promising solutions to this problem is device-to-device (D2D) communication system [1-3]. D2D communication system is one of the standards of the 5th generation (5G) cellular communication system. D2D communication is defined as the high data rate direct communication between two users' equipment with high spectral efficiency, without passing through cellular infrastructures [4-6]. Presence of large number of various types of wireless devices as well as D2D pairs sharing a cellular communication spectrum can cause unwanted and unintentional co-channel interference (CCI) in the absence of proper coordination. Therefore, CCI should be considered while analyzing the performance of D2D communication systems [7-8]. Outage probability, channel capacity and symbol-error rate (SER) are well-known parameters used for the performance analysis of the wireless communication systems. In [9], authors analyzed outage probability of D2D communication systems over Rayleigh fading channels. The outage probability of D2D communication system under Suzuki fading in presence of co-channel interference is studied in [10]. Channel capacity of a D2D system, over a Rician faded channel, is analyzed in [11]. Authors in [12], studied D2D channel capacity over a correlated Rayleigh fading channel in an interference limited environment. In [13], authors presented a study of SER of a multicarrier D2D video transmission over an independent Rayleigh fading channel.

In this paper, outage probability, channel capacity and SER of a D2D communication system are investigated. A D2D pair is assumed to be affected by various co-channel interferers in the system. The CCI may arise from any wireless or D2D communication device in the system when proper coordination is lost or interrupted. The fading channel for the desired D2D system is considered to be double-generalized gamma (dGG). The dGG is a versatile and mathematically tractable fading distribution. The dGG variable is a product of two generalizedGamma variables [14]. The dGG includes various distributions like Gamma-Gamma, double-Nakagami, double-Weibull, Weibull-Gamma, and double-Rayleigh. It can also model distributions like, generalized-Gamma and Nakagami/Rayleigh -lognormal. In cellular communication systems, signals are often scattered and re-scattered around transmitter's and receiver's local surroundings. This double-scattering channel condition is observed when the transmitter and receiver devices are moving. The double-scattering conditions can be effectively modeled with the help of dGG distribution [15]. The propagation channel distribution for the CCI is assumed to be Nakagami. Which is a well-known versatile distribution often used in the literature to model various channel fading conditions. The rest of this paper is organized as follows. Section II presents the D2D system model and the expressions for the outage probability, channel capacity and SER. The numerical results are discussed in Section III and Section IV concludes the paper.

\section{SYSTEM MODEL}

D2D communication network having $N$ interferers is shown in Figure 1. In this work, a co-channel interferer is assumed to be any wireless node or any D2D communication device. A D2D pair is considered here for the performance analysis. This 
D2D pair is termed as desired or considered D2D pair. There can be many small objects in the transmitter and receiver environment of a D2D pair which can cause double scattering. The dGG distribution can effectively model double scattering. The channel for the considered D2D pair is assumed to be dGG. The channel for the $i$-th interferer to the desired D2D pair's receiver is assumed to be Nakagami distributed. The system is considered to be interference limited [16]. In order to keep the analytical work tractable and to obtain easy-tocompute mathematical expressions, the following assumptions are adopted: 1) Co-channel interferers are located at approximately the same distance from the desired D2D pair's receiver. 2) Co-channel interferers are assumed to be independent and identically distributed.

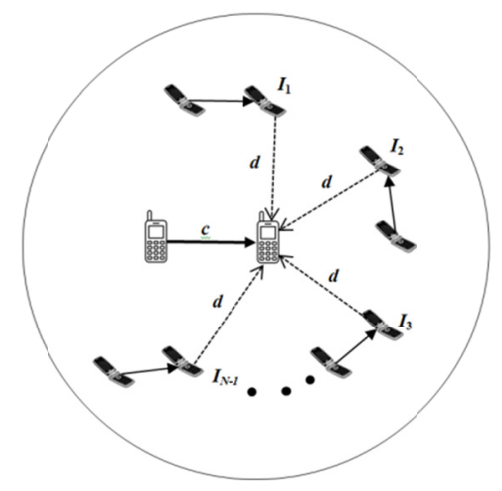

Fig. 1. System model

TABLE I. NOMENCLATURE

\begin{tabular}{|c|c|}
\hline Considered or desired D2D pair device & \\
\hline$i$-th interferer & $\mathrm{c}$ \\
\hline Distance between the devices of the desired D2D pair & $\mathrm{d}$ \\
\hline Distance between i-th interferer and the desired D2D pair's receiver & $\longrightarrow$ \\
\hline Desired D2D pair communication signal & \\
\hline $\begin{array}{c}\text { Interference signal from i-th interferer to the receiver of the desired } \\
\text { D2D pair }\end{array}$ & $-\cdots$ \\
\hline Communication signals for other devices & $\longrightarrow$ \\
\hline
\end{tabular}

The PDF of dGG distribution is [15]:

$$
f_{Y}(y)=\frac{2 \beta(\omega)^{\frac{\delta_{1}+\delta_{2}}{2}}}{\Gamma\left(\delta_{1}\right) \Gamma\left(\delta_{2}\right)} y^{\frac{\beta}{2}\left(\delta_{1}+\delta_{2}\right)-1} K_{\delta_{2}-\delta_{1}}\left(2 \sqrt{\omega} y^{\frac{\beta}{2}}\right), \quad y>0
$$

where $\delta_{1}, \delta_{2}$ and $\beta$ are the fading shape parameters that imply the severity of the fading conditions, $\omega=\delta_{1} \delta_{2} / \varepsilon_{1} \varepsilon_{2}$ where $\varepsilon_{1}$ and $\varepsilon_{2}$ are related to average power of fading and $K_{v}(z)$ is the modified Bessel function of the second kind [17]. The PDF of the Nakagami distribution is [18

$$
f_{X}(x)=\frac{2}{\Gamma(\mu)}\left(\frac{\mu}{\ell}\right)^{\mu} x^{2 \mu-1} \exp \left(\frac{-\mu}{\ell} x^{2}\right), \quad \mu \geq \frac{1}{2}, \ell>0, x>0
$$

where $\mu$ is the fading parameter which describes the severity of the channel fading conditions, $\ell$ is the average power of fading and $\Gamma($.$) is gamma function [19]. Path-loss is an$ important factor that also affects the performance of a wireless communication system. A simplified path-loss model is considered here [20]. The power at the desired D2D receiver's pair is:

$$
S_{d}=P_{D}\left(\frac{\lambda}{4 \pi c_{0}}\right)^{2}\left(\frac{c_{0}}{c}\right)^{a}
$$

where $P_{D}$ is the D2D signal power, $c$ is the distance between the considered D2D pair devices, $\lambda$ is the wavelength, $\alpha$ is the path-loss exponent $(2 \leq \alpha \leq 5)$ and $c_{0}$ is the reference distance $(1 \mathrm{~m}$ to $100 \mathrm{~m})$. Similarly, the power of $i$-th interferer at the receiver of the desired D2D pair is:

$$
I=P_{I}\left(\frac{\lambda}{4 \pi d_{0}}\right)^{2}\left(\frac{d_{0}}{d}\right)^{b} .
$$

In (2), $P_{I}$ is the interference signal power, $d$ is the distance between the receiver of the considered D2D pair and the $i$-th interferer, $b$ is the path-loss exponent $(2 \leq b \leq 5)$ of the interference signal and $d_{0}$ is the reference distance $(1 \mathrm{~m}$ to $100 \mathrm{~m})$. Generally, in this work $P_{D}$ and $P_{I}$ are not considered to be equal. Based on (1) and (2), the desired signal power to the interference signal power ratio (SIR) of the D2D system is:

$$
\gamma=\frac{g^{2}}{h \sum_{i=1}^{N} \alpha_{i}^{2}}, \quad h=\frac{P_{D}}{P_{I}}\left(\frac{c^{a}}{d^{b}}\right) \frac{\left(c_{0}\right)^{2-a}}{\left(d_{0}\right)^{2-b}}
$$

where $g$ and $\alpha_{i}$ are the independent dGG fading variable of the desired D2D pair's signal and independent Nakagami fading variable of the $i$-th interferer, respectively. $N$ is the number of interferers in the system. The SIR PDF expression of the D2D system, $f_{\gamma}(r)$, can be found by using the identity,

$$
\begin{aligned}
f_{\gamma}(r)=\int_{0}^{\infty} x f_{S}(r x) f_{I}(x) d x,[21] \\
f_{\gamma}(r)=\int_{0}^{\infty} x \underbrace{\frac{\beta \omega^{\frac{\delta_{1}+\delta_{2}}{2}}(r x)^{\frac{\beta}{4}\left(\delta_{1}+\delta_{2}\right)-1} K_{\delta_{2}-\delta_{1}}\left(2 \sqrt{\omega}(r x)^{\frac{\beta}{4}}\right)}{2 \Gamma\left(\delta_{1}\right) \Gamma\left(\delta_{2}\right)}}_{f_{S}(r x)} \\
\times \underbrace{\frac{\Omega^{m_{T}} x^{m_{T}-1}}{\Gamma\left(m_{T}\right)} \exp (-\Omega x)}_{f_{I}(x)} d x, \\
\left.f_{\gamma}(r)=\sigma(r)^{\frac{\beta}{4}\left(\delta_{1}+\delta_{2}\right)-1} G_{\beta, 4}^{4, \beta}\left[\left(\frac{\omega}{4}\right)^{2}\left(\frac{r \beta}{\Omega}\right)^{\beta} \mid \boldsymbol{\Delta}_{1}\right] \boldsymbol{\Delta}_{2}\right]
\end{aligned}
$$

where $\beta$ a positive integer, $\delta_{1}$ and $\delta_{2}$ are the dGG distribution shape parameters of the desired $\mathrm{D} 2 \mathrm{D}, \omega$ is related to the average power of the dGG fading channel. Parameter $m_{T}=N m$, where $m$ is the shape parameter of the interference signal, parameter $\Omega$ is given as $\Omega=\varphi / h$ where $\varphi=m / \eta$ in which $\eta$ is the average power of the interference fading channel. Moreover,

$$
\sigma=\frac{\omega^{\frac{\delta_{1}+\delta_{2}}{2}} \beta^{\frac{\beta}{4}\left(\delta_{1}+\delta_{2}\right)+m_{T}+\frac{1}{2}} \Omega^{-\frac{\beta}{4}\left(\delta_{1}+\delta_{2}\right)}}{2 \Gamma\left(\delta_{1}\right) \Gamma\left(\delta_{2}\right) \Gamma\left(m_{T}\right)(2 \pi)^{\frac{\beta+1}{2}}}
$$




$$
\begin{aligned}
& \boldsymbol{\Delta}_{1}=\frac{1-\frac{\beta}{4}\left(\delta_{1}+\delta_{2}\right)-m_{T}}{\beta}, \cdots, \frac{\beta-\frac{\beta}{4}\left(\delta_{1}+\delta_{2}\right)-m_{T}}{\beta} \quad \text { and } \\
& \boldsymbol{\Delta}_{2}=\frac{\delta_{2}-\delta_{1}}{4}, \frac{\delta_{2}-\delta_{1}+2}{4}, \frac{\delta_{1}-\delta_{2}}{4}, \frac{\delta_{1}-\delta_{2}+2}{4}
\end{aligned}
$$

With the help of (4), the cumulative distribution function (CDF) of SIR of the D2D system is [22]

$$
F_{\gamma}(r)=\sigma \beta^{-1}(r)^{\frac{\beta}{4}\left(\delta_{1}+\delta_{2}\right)} G_{2 \beta, 4+\beta}^{4,2 \beta}\left[\left(\frac{\omega}{4}\right)^{2}\left(\frac{r \beta}{\Omega}\right)^{\beta} \mid \begin{array}{c}
\boldsymbol{\Delta}_{3}, \boldsymbol{\Delta}_{1} \\
\boldsymbol{\Delta}_{2}, \boldsymbol{\Delta}_{4}
\end{array}\right]
$$

where:

$$
\begin{aligned}
& \Delta_{3}=\frac{1-\frac{\beta}{4}\left(\delta_{1}+\delta_{2}\right)}{\beta}, \cdots, \frac{\beta-\frac{\beta}{4}\left(\delta_{1}+\delta_{2}\right)}{\beta}, \\
& \Delta_{4}=-\frac{\left(\delta_{1}+\delta_{2}\right)}{4}, \cdots, \frac{\beta-\frac{\beta}{4}\left(\delta_{1}+\delta_{2}\right)-1}{\beta} .
\end{aligned}
$$

The probability of dropping of the SIR of a system below a predefined threshold $R$ is defined as the outage probability. By using $P_{\text {out }}=\int_{0}^{R} f_{\gamma}(r) d r$, the expression for the outage probability of the D2D system is [22]:

$$
P_{\text {out }}=\sigma \beta^{-1}(R)^{\frac{\beta}{4}\left(\delta_{1}+\delta_{2}\right)} G_{2 \beta, 4+\beta}^{4,2 \beta}\left[\left(\frac{\omega}{4}\right)^{2}\left(\frac{r \beta}{\Omega}\right)^{\beta} \mid \begin{array}{l}
\boldsymbol{\Delta}_{3}, \boldsymbol{\Delta}_{1} \\
\boldsymbol{\Delta}_{2}, \boldsymbol{\Delta}_{4}
\end{array}\right]
$$

Now, the expression for channel capacity of the D2D communication system is obtained by using the formula $C=\int_{0}^{\infty} \log _{2}(1+r) f_{\gamma}(r) d r$, as in [23]:

$$
C=\frac{\sigma \beta^{-1}}{\ln (2)(2 \pi)^{\beta-1}} G_{2 \beta, 4+2 \beta}^{4+2 \beta, 2 \beta}\left[\left(\frac{\omega}{4}\right)^{2}\left(\frac{\beta}{\Omega}\right)^{\beta} \mid \begin{array}{l}
\Delta_{1}, \Delta_{5}, \Delta_{3} \\
\Delta_{2}, \Delta_{5}, \Delta_{5}
\end{array}\right]
$$

where $\Delta_{5}=\frac{-\frac{\beta}{4}\left(\delta_{1}+\delta_{2}\right)}{\beta}, \cdots, \frac{\beta-\frac{\beta}{4}\left(\delta_{1}+\delta_{2}\right)-1}{\beta}$.

SER expression of the D2D communication system is now presented. $M$-ary phase-shift keying ( $M$-PSK) based modulation scheme is incorporated in this work. With the help of [22-24] the SER expression is

$$
S E R=\rho \int_{0}^{\left(\frac{M-1}{M}\right)^{\pi}} G_{2 \beta, 4}^{4,2 \beta}\left[\left.\left(\frac{\omega}{4}\right)^{2}\left(\frac{\beta}{\Omega}\right)^{\beta}\left(\frac{\sin (\theta)}{\sin \left(\frac{\pi}{M}\right)}\right)^{2 \beta}\right|_{\boldsymbol{\Delta}_{7}}\right] d \theta
$$

where $\rho=\frac{(\omega)^{\frac{\delta_{1}+\delta_{2}}{2}}(\Omega)^{-\frac{\beta}{4}\left(\delta_{1}+\delta_{2}\right)}(\beta)^{\frac{\beta}{2}\left(\delta_{1}+\delta_{2}\right)+m_{T}}}{\Gamma\left(\delta_{1}\right) \Gamma\left(\delta_{2}\right) \Gamma\left(\delta_{m_{T}}\right)(2 \pi)^{\beta+1}}$,

$$
\Delta_{6}=\frac{1}{\beta}, \cdots, 1, \frac{1-m_{T}}{\beta}, \cdots \frac{\beta-m_{T}}{\beta} \text { and }
$$

$$
\boldsymbol{\Delta}_{7}=\frac{\delta_{2}}{2}, \cdots, \frac{1+\delta_{2}}{2}, \frac{\delta_{1}}{2}, \cdots, \frac{1+\delta_{1}}{2}
$$

Expressions of the outage probability, capacity and SER are valid for arbitrary parameters of $\mathrm{dGG}$ and Nakagami distribution, path-loss and co-channel interference.

\section{NUMERICAL ANALYSIS AND RESULTS}

In this Section numerical results are presented and discussed. For analysis simplicity the reference distances $c_{0}$ and $d_{0}$ are assumed to be $1 \mathrm{~m}$. The SIR threshold $R$ is set at $10 \mathrm{dBm}$. Outage performance of D2D system with varying values of two of shape parameters i.e. $\delta_{1}$ and $\delta_{2}$ is shown in Figure 2. Transmit power $P_{D}$, path-loss exponent $a$, and shape parameter $\beta$ are fixed at $20 \mathrm{dBm}, 3$ and 2 , respectively. Power $P_{I}$, path-loss exponent $b$, the number of interferers $N$, the distance between the $i$-th interferer and the receiver of the desired D2D pair $d$, and the fading parameter $m$ are assumed to be $10 \mathrm{dBm}, 3.5,5$, $50 \mathrm{~m}$ and 2 , respectively. It is observed that the fading conditions of the D2D system improve with the increase in the values of fading parameters, i.e. $\delta_{1}$ and $\delta_{2}$. A degradation in the outage performance of D2D system is also observed for the similar channel fading conditions of the desired D2D pair when the value of $c$ is increased. As the desired D2D pair's transmitter and receiver move away from each other, i.e. the value of $c$ increases, outage performance of the D2D system degrades because of the power decreasing of the desired D2D signal caused by the path-loss effects.



Fig. 2. Outage performance with varying values of two of shape parameters.

The outage performance comparison of the D2D communication system for various values of dGG fading parameter, $\beta$ and the path-loss exponent $a$ is shown in Figure 3 . The values for the desired signal parameters $P_{D}, \delta_{1}, \delta_{2}$ and $c$ are fixed at $20 \mathrm{dBm}, 1.5,2$ and $25 \mathrm{~m}$, respectively. The values of interference parameters $P_{I}, b, N, d, m$ are assumed to be $10 \mathrm{dBm}, 4,5,60 \mathrm{~m}$ and 1 , respectively. It is observed that the outage performance of the D2D system is improved for the higher values of dGG shape parameter $\beta$ of the desired signal. This happens because of the improved fading conditions with the increase in the values of the desired signal's shape parameter. Moreover, for the same values of fading shape parameter of the desired signal, outage performance deteriorates with increase in path-loss exponent $a$ because of the worsened SIR condition of the system when the value of $a$ is increased. 


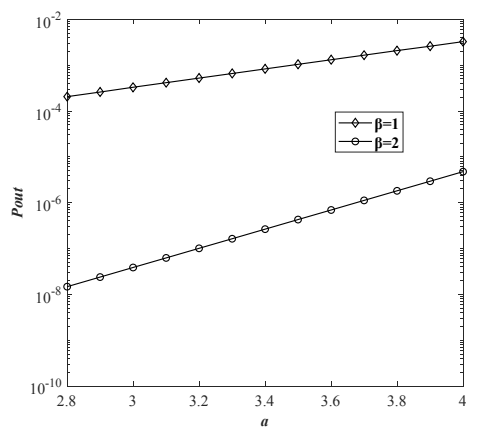

Fig. 3. Outage performance with varying values of fading parameter $\beta$.

Outage performance of the D2D system for various values of path-loss exponent of the interference signal $b$ is shown in Figure 4. For the desired signal the values of $P_{D}, a, \beta, \delta_{1}$ and $\delta_{2}$ are set to be $20 \mathrm{dBm}, 2.5,2,2$ and 1 , respectively. For the interference signals $P_{I}, m, N$ and $d$ are assumed to be $10 \mathrm{dBm}, 2$, 5 and $50 \mathrm{~m}$, respectively. It is noticed that the outage performance is better for the higher values of $b$ because of the weakening of the interference signals at the desired D2D pair's receiver when the value of the CCI path-loss exponent $b$ is increased. Hence, an improved outage performance of the D2D system is observed. It is also observed that as the desired D2D pair's devices move away from each other, the value of $c$ increases and outage performance of the D2D system deteriorates because of the weakening of the desired D2D signal caused by the path-loss effects.

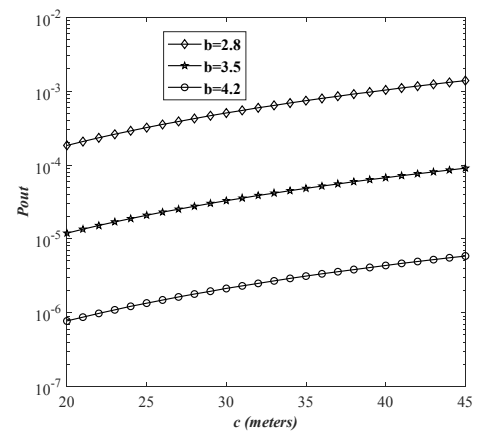

Fig. 4. Outage performance with various values of path-loss exponent $b$

Figure 5 illustrates the outage performance with various values of the interference fading shape parameter $m$ and distance $d$. The values of $P_{D}, P_{I}, a, \beta, \delta_{1}, \delta_{2}, b, N$ and $c$ are fixed at $20 \mathrm{dBm}, 10 \mathrm{dBm}, 2.5,2,1.5,3,3$ and $25 \mathrm{~m}$, respectively. It is observed that the system outage performance is almost insensitive to the interference fading conditions. Moreover, for the same value of $m$ it is observed that the outage performance of the system improves with increasing values of the distance $d$, because as the interferers move away from the desired D2D pair's receiver the interference signal strength weakens due to path-loss effects.

Channel capacity performance of D2D communication system with varying values of $N$ and the path-loss exponent $a$ is shown in Figure 6. The values of $P_{D}, P_{I}, m, \beta, \delta_{1}, \delta_{2}, b, c$ and $d$ are set to be $20 \mathrm{dBm}, 10 \mathrm{dBm}, 2,1,1,2,3,30 \mathrm{~m}$ and $60 \mathrm{~m}$, respectively. It is clear that the channel capacity performance of the system is better when the number of interferers $N$ is decreased. It is also observed that as the path-loss exponent value of the desired signal is increased, the capacity performance of the system degrades due to the weakening of the desired signal strength due to path-loss effects.

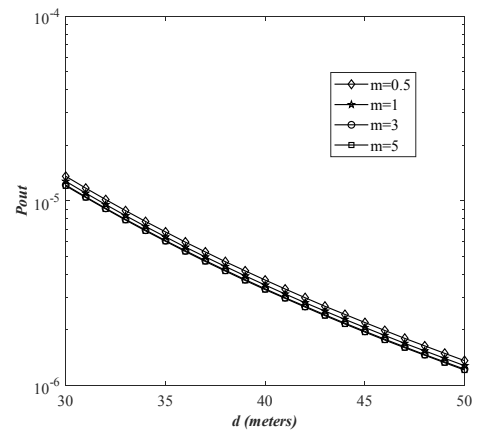

Fig. 5. Outage performance with various values of the shape parameter of $\mathrm{CCI}$

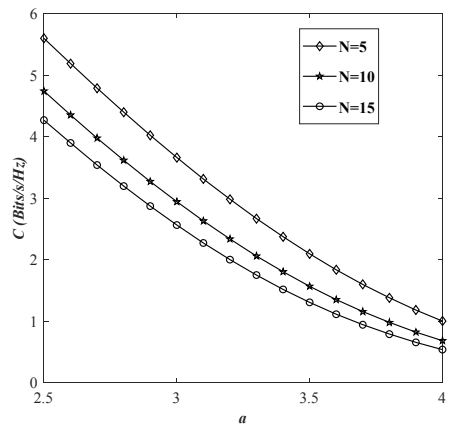

Fig. 6. Channel capacity performance of D2D communication system for various $N$

Figure 7 shows the channel capacity performance of the D2D system for different values of distances $d$ and $c$. The values of $P_{D}, P_{I}, a, b, m, \beta, \delta_{1}, \delta_{2}$ and $N$ are assumed to be $20 \mathrm{dBm}, 10 \mathrm{dBm}, 3,3.3,2,1,1,2$ and 5 , respectively. it is observed that the channel capacity is improved for higher values of the distance $d$ because of the improvement of the SIR conditions due to weakening of the interference signals at the desired D2D pair's receiver.



Fig. 7. Capacity performance of D2D communication system with varying distance $d$ 
SER performance of 8-PSK scheme with various values of number of interference signals is shown in Figure 8. The values of $P_{D}, P_{I}, \beta, \delta_{1}, \delta_{2}, m, a, c$ and $d$ are considered to be $20 \mathrm{dBm}$, $10 \mathrm{dBm}, 1,2,3,2,3.7,20 \mathrm{~m}$ and $60 \mathrm{~m}$ respectively. The SER performance improves when the number of interferers is reduced. Moreover, for the same number of interferers, SER performance improves with an increase in the values of interferers' path-loss exponent $b$.

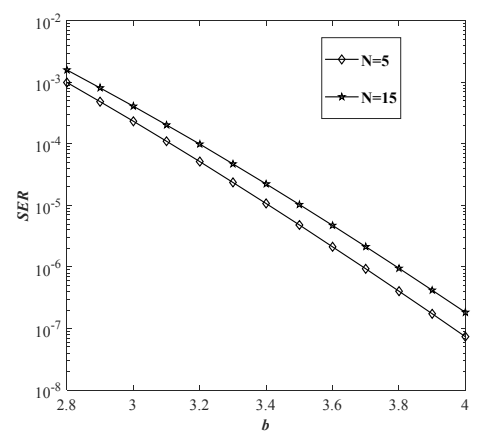

Fig. 8. SER performance with various values of number of CCIs

\section{CONCLUSION}

In this paper, the performance of a D2D communication system over a dGG faded channel is analyzed. The dGG distribution is a general distribution that includes all others that are used to model double-scattering which occur in wireless communication systems. Effects of co-channel interference caused by various wireless devices in the system are included. Expressions of the PDF and CDF of the SIR of the D2D system are presented. Based on the PDF of the SIR, expressions of the performance metrics like outage probability, channel capacity and SER are presented. Based on these expressions, numerical results are presented to discuss the performance of the D2D system under varying interference, path-loss and channel fading conditions. It is observed that the path-loss significantly affects the performance of the D2D communication system. Moreover, co-channel interference, regardless of being affected by the channel fading and path-loss conditions, manages to affect the performance of the D2D system.

\section{REFERENCES}

[1] M. Waqas, M. Ahmed, Y. Li, D. Jin, S. Chen, "Social-Aware Secret Key Generation for Secure Device-to-Device Communication via Trusted and Non-Trusted Relays", IEEE Transactions on Wireless Communications, Vol. 17, No. 6, pp. 3918-3930, 2018

[2] J. Z. Moghaddam, M. Usman, F. Granelli, "A Device-to-Device Communication-Based Disaster Response Network", IEEE Transactions on Cognitive Communications and Networking, Vol. 4, No. 2, pp. 288298, 2018

[3] J. H. Kim, J. Joung, J. W. Lee, "Resource Allocation for Multiple Device-to-Device Cluster Multicast Communications Underlay Cellular Networks", IEEE Communications Letters, Vol. 22, No. 2, pp. 412-415, 2018

[4] Y. J. Chun, G. B. Colombo, S. L. Cotton, W. G. Scanlon, R. M. Whitaker, S. M. Allen, "Device-to-Device Communications: A Performance Analysis in the Context of Social Comparison-Based Relaying", IEEE Transactions on Wireless Communications, Vol. 16, No. 12, pp. 7733-7745, 2017

[5] K. Yang, S. Martin, C. Xing, J. Wu and R. Fan, "Energy-Efficient Power Control for Device-to-Device Communications", IEEE Journal on
Selected Areas in Communications, Vol. 34, No. 12, pp. 3208-3220, 2016

[6] J. Wang, Q. Tang, C. Yang, R. Schober, J. Li, "Security Enhancement via Device-to-Device Communication in Cellular Networks", IEEE Signal Processing Letters, Vol. 23, No. 11, pp. 1622-1626, 2016

[7] Y. J. Chun, S. L. Cotton, H. S. Dhillon, A. Ghrayeb, M. O. Hasna, "A Stochastic Geometric Analysis of Device-to-Device Communications Operating Over Generalized Fading Channels", IEEE Transactions on Wireless Communications, Vol. 16, No. 7, pp. 4151-4165, 2017

[8] K. Yang, S. Martin, C. Xing, J. Wu, R. Fan, "Energy-Efficient Power Control for Device-to-Device Communications", IEEE Journal on Selected Areas in Communications, Vol. 34, No. 12, pp. 3208-3220, 2016

[9] S. Li, Q. Ni, Y. Sun, G. Min, "Resource Allocation for Weighted SumRate Maximization in Multi-User Full-Duplex Device-to-Device Communications: Approaches for Perfect and Statistical CSIs", IEEE Access, Vol. 5, pp. 27229-27241, 2017

[10] H. Ghavami, S. Shirvani Moghaddam, "Outage Probability of Device to Device Communications Underlaying Cellular Network in Suzuki Fading Channel", IEEE Communications Letters, Vol. 21, No. 5, pp. 1203-1206, 2017

[11] C. Liu, B. Natarajan, "Power-Aware Maximization of Ergodic Capacity in D2D Underlay Networks", IEEE Transactions on Vehicular Technology, Vol. 66, No. 3, pp. 2727-2739, 2017

[12] H. Min, J. Lee, S. Park, D. Hong, "Capacity Enhancement Using an Interference Limited Area for Device-to-Device Uplink Underlaying Cellular Networks", IEEE Transactions on Wireless Communications, Vol. 10, No. 12, pp. 3995-4000, 2011

[13] P. Wu, P. C. Cosman, L. B. Milstein, "Resource Allocation for Multicarrier Device-to-Device Video Transmission: Symbol Error Rate Analysis and Algorithm Design", IEEE Transactions on Communications, Vol. 65, No. 10, pp. 4446-4462, 2017

[14] P. S. Bithas, A. G. Kanatas, D. B. da Costa, P. K. Upadhyay, U. S. Dias, "On the Double-Generalized Gamma Statistics and Their Application to the Performance Analysis of V2V Communications", IEEE Transactions on Communications, Vol. 66, No. 1, pp. 448-460, 2018

[15] P. S. Bithas, A. G. Kanatas, D. B. da Costa, P. K. Upadhyay, U. S. Dias, "The Double-Generalized Gamma Distribution and Its Application to V2V Communications", GLOBECOM 2017-IEEE Global Communications Conference, Singapore, December 4-8, 2017

[16] S. Parthasarathy, R. K. Ganti, "Impact of Shadowing in D2D Communication", 2017 IEEE Wireless Communications and Networking Conference, San Francisco, USA, March 19-22, IEEE, 2017

[17] Wolfram Research, BesselK, Modified Bessel Function of the Secong Kind, Available at: http://functions.wolfram.com/Bessel-TypeFunctions /BesselK/02/ [Last Accessed July 2018]

[18] A. Mehbodniya, S. Aissa, "Outage and BER Analysis for Ultrawideband-Based WPAN in Nakagami-m Fading Channels", IEEE Transactions on Vehicular Technology, Vol. 60, No. 7, pp. 3515-3520, 2011

[19] I. S. Gradshteyn, I. M. Ryzhik, Table of Integrals, Series, and Products, Academic Press, 2007

[20] A. Goldsmith, Wireless Communications, Cambridge University Press, 2005

[21] I. Trigui, A. Laourine, S. Affes, A. Stephenne, "Performance analysis of mobile radio systems over composite fading/shadowing channels with co-located interference", IEEE Transactions on Wireless Communications, Vol. 8, No. 7, pp. 3448-3453, 2009

[22] Wolfram Research, MeijerG, Meijer G-Function, available at: http:// functions.wolfram.com/HypergeometricFunctions/MeijerG/21/

[23] M. S. Alouini, A. J. Goldsmith, "Capacity of Rayleigh fading channels under different adaptive transmission and diversity-combining techniques", IEEE Transactions on Vehicular Technology, Vol. 48, No. 4, pp. 1165-1181, 1999

[24] B. Barua, M. Abolhasan, D. R. Franklin, F. Safaei, "SEP of Multihop Relay Networks in Nakagami-m Fading Channels", IEEE 78th Vehicular Technology Conference, Las Vegas, USA, September 2-5, 2013 